\title{
Integrating abortion into Canada's primary health care system with mifepristone
}

\author{
Grady Arnott, Grace Sheehy, and Kathryn J. LaRoche
}

\section{University of 0ttawa}

Although the majority of abortions worldwide are performed surgically, termination of pregnancy using medication (termed "medication abortion" in reproductive health fields $)^{1}$ is becoming more common due to its costeffectiveness, accessibility, and desirability among patients. Mifepristone, also known as RU486, is an anti-progesterone steroid, and it is considered the gold standard of medication abortion. ${ }^{2}$ This medication is taken in the form of a tablet and is effective for early abortion up through 63 days (nine weeks) after the start of the last menstrual period. When mifepristone is used in conjunction with misoprostol, a medication that induces uterine contractions, the regimen results in a complete abortion $95-98 \%$ of the time. ${ }^{2}$ Despite this regimen being the international gold standard, mifepristone is currently not registered in Canada and is therefore unavailable for commercial distribution in the country. However, early 2014 was met with national media reports and discussions within the published literature about a pending Health Canada application to register the drug. ${ }^{3}$ In the midst of awaiting a decision from Health Canada, it is important to consider the potential role for mifepristone in Canada's healthcare system. Mifepristone could increase access to abortion at the primary health care level due to the fact that it can be prescribed and administered by a variety of providers. ${ }^{4}$ Therefore, mifepristone's registration in Canada should be of tremendous interest to primary health care practitioners and patients.

Canada's primary health care system is currently experiencing a pivotal moment of transformation; increases in patient-centered care, expanded and improved training programs, and task sharing within interprofessional teams are reshaping the way we conceptualize and deliver primary health care. ${ }^{5,6}$ Since 1997 , the number of primary health care providers in Canada who are able to provide reproductive health services, including physicians, nurse practitioners, and midwives has grown. ${ }^{6}$ Physicians and nurse practitioners are increasingly being trained in the prescription and administration of medication previously only available through specialized providers; mifepristone could be included in this scope. Counselling women on their pregnancy options, determining gestational age, and providing medication abortion and post-abortion care all fall within the scope of primary care clinicians' practice. ${ }^{4} \mathrm{~A}$ 2002 study found that Canadian family medicine physicians and residents expressed an interest in receiving more information about medication abortion. More than half of physicians said they would consider providing medication abortion care to their patients. ${ }^{7}$

If primary health care providers can provide medication abortion using mifepristone in their practices, abortion may become more accessible to women across Canada. Women in Canada are reported to travel long distances and experience wait times when seeking abortion care; one survey found that $73.5 \%$ of women at a Toronto abortion clinic travelled one hour or more to access care. ${ }^{8}$ An increase in the number of primary providers who can potentially provide abortion care means more avenues through which abortion options are shared with patients, thus empowering women to have greater control and input into their own reproductive health. By increasing the provision of reproductive health services through primary health care providers, women have more opportunities to become engaged in their reproductive health and able to seek abortion care with fewer travels and delays.

Bringing abortion into the offices of primary health care providers offers an opportunity for knowledge transfer and reduced stigma. Due to an established relationship and comfort with their family physician, provision of medication abortion within the family health care unit is highly acceptable among female patients. ${ }^{9} \mathrm{~A}$ model that encourages knowledge transfer between provider and female patients about abortion options within familiar 
health settings allows women to make informed decisions with a trusted provider with whom they have an existing relationship. Historically, the fact that abortion care has been relegated to a specialized setting has contributed to stigmatization of the procedure and patients. Since the public discourse on abortion can be entrenched in polemic, integrating the procedure into a familiar environment at the primary health care level may reduce the stigma women face when accessing care.

One in three Canadian women will choose to terminate a pregnancy in their lifetimes. ${ }^{10}$ If mifepristone is registered in Canada, it will open a new channel of choice for patients; it also has the potential to expand access to safe, high quality services. Reframing abortion within primary health care could make it more accessible to Canada's diverse population, simultaneously reducing the stigma associated with the procedure by bringing it into doctor's offices and women's homes. Through shared knowledge between primary care providers and patients, women seeking abortion care would be empowered to make educated decisions rooted in sound evidence, thus leading to more optimal reproductive health outcomes for women across Canada.

\section{References}

1. Weitz TA, Foster A, Ellertson C, Grossman D, Stewart FH. "Medical" and "surgical" abortion: Rethinking the modifiers. Contraception. 2004;69(1):77-8.

2. Department of Reproductive Health and Research, World Health Organization (WHO). Safe abortion: Technical and policy guidance for health systems. 2nd ed. Geneva: WHO; 2012 [updated 2012 Jun 19, cited 2014 Feb 12]. Available from: http://apps.who.int/iris/ bitstream/10665/70914/1/9789241548434 eng.pdf

3. Dunn S, Cook R. Medical abortion in Canada: Behind the times. CMAJ. 2014;186(1):13-4.

4. Yanow S. It is time to integrate abortion into primary care. Am J Public Health. 2013;103(1):14-6.

5. Hutchison B, Levesque JF, Strumpf E, Coyle N. Primary health care in Canada: Systems in motion. Milbank Q. 2011;89(2):256-88.

6. Canadian Institute for Heath Information (ClHI). Canada's Health Care Providers, 1997 to 2011: A reference guide. Ottawa: $\mathrm{ClHI} ; 2013$ [last update unknown; cited 2014 Feb 12]. Available from: http://publications.gc.ca/ collections/collection_2013/icis-cihi/H118-1-4-2011-eng.pdf

7. Raymond E, Kaczorowski J, Smith P, Sellors J, Walsh A. Medical abortion and family physicians. Survey of residents and practitioners in two Ontario settings. Can Fam Physician. 2002;48:538-44.

8. Sethna S, Doull M. Far from home? A pilot study tracking women's journey's to an abortion clinic. J Obstet Gynaecol Can. 2007;27(8):640-64.

9. Rubin SE, Godfrey EM, Shapiro M, Gold M. Urban female patients' perceptions of the family medicine clinic as a site for abortion care. Contraception. 2009;80(2):174-9.

10. Norman WV. Induced abortion in Canada 1974-2005: Trends over the first generation with legal access. Contraception. 2012;85(2):185-91.

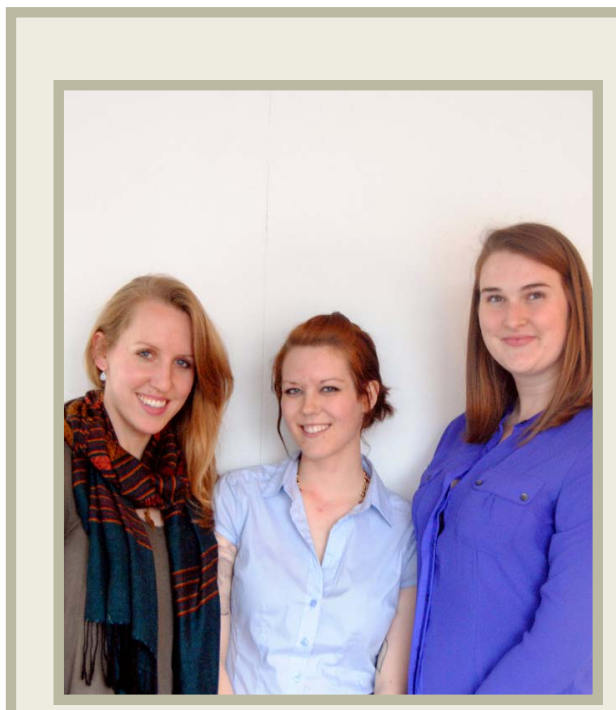

\section{Grady Arnott}

Grady Arnott is an MSc Candidate in the Interdisciplinary School of Health Sciences at the University of Ottawa. Her multi-methods research explores the implications of health policy on access to reproductive health commodities and programming in refugee, conflict, crisis, and emergency settings. Her research interests include reproductive health and justice, and action-oriented policy research.

\section{Grace Sheehy}

Grace Sheehy is a Master's student in the Interdisciplinary Health Sciences program at the University of Ottawa. Her thesis is a qualitative study of reproductive health in Yangon, Myanmar. Through focus group discussions, key informant interviews, and service mapping, she will document women's reproductive health experiences, service accessibility, and avenues for improving service delivery. Her research interests are in sexual and reproductive health, both in Canada and abroad.

\section{Kathryn J. LaRoche}

Kathryn J. LaRoche is completing her Masters in Interdisciplinary Health Sciences at the University of Ottawa. She is interested in action-oriented, qualitative research related to sexual health. More specifically, her work has focused on exploring issues of abortion access and post-abortion support for women in North America. Her thesis is an in-depth exploration of post-abortion support in Ontario. 